\title{
Calculation of exchange energies using algebraic perturbation theory
}

\author{
B. L. Burrows, ${ }^{1, *}$ A. Dalgarno, ${ }^{2, \dagger}$ and M. Cohen ${ }^{3, \ddagger}$ \\ ${ }^{1}$ Mathematics Section Faculty of Computing, Engineering and Technology Staffordshire University, Beaconside, \\ Stafford ST18 ODG, United Kingdom \\ ${ }^{2}$ Harvard-Smithsonian Center for Astrophysics, 60 Garden Street, Cambridge, Massachusetts 02138, USA \\ ${ }^{3}$ Department of Physical Chemistry, The Hebrew University of Jerusalem, Jerusalem IL-91904, Israel
}

(Received 10 February 2010; published 14 April 2010)

\begin{abstract}
An algebraic perturbation theory is presented for efficient calculations of localized states and hence of exchange energies, which are the differences between low-lying states of the valence electron of a molecule, formed by the collision of an ion $Y^{+}$with an atom $X$. For the case of a homonuclear molecule these are the gerade and ungerade states and the exchange energy is an exponentially decreasing function of the internuclear distance. For such homonuclear systems the theory is used in conjunction with the Herring-Holstein technique to give accurate exchange energies for a range of intermolecular separations $R$. Since the perturbation parameter is essentially $1 / R$, this method is suitable for large $R$. In particular, exchange energies are calculated for $X_{2}{ }^{+}$systems, where $X$ is $\mathrm{H}, \mathrm{Li}, \mathrm{Na}, \mathrm{K}, \mathrm{Rb}$, or $\mathrm{Cs}$.
\end{abstract}

DOI: 10.1103/PhysRevA.81.042508

PACS number(s): $31.15 . \mathrm{B}-, 31.15 .-\mathrm{p}, 31.10 .+\mathrm{z}$

\section{INTRODUCTION}

The problem of exchange energies between two low-lying states of a two-atom ionic system is considered. Initially, we examine the case where the atoms are hydrogenlike atoms separated by an internuclear distance $R$, which we then generalize to larger atoms, each with a predominant valence electron which is described in the same formalism. The solution of the nonrelativistic Schrödinger equation obtained is perturbative, based on the exact solutions at infinite separation; for the larger atoms, these are described by the asymptotic valence states determined by the ionization energies of the atoms. The perturbation can conveniently be described in an algebraic formalism but it follows the well-known technique of finding a perturbation expansion for the separation constant of the Schrödinger equation when spheroidal coordinates are used. The perturbation parameter is a function of both $R$ and the energy $E$ of the state considered, and $E$ is determined by equating the expansions of the separation constant from each of the separated equations.

Initially, we concentrate on heteronuclear molecules but there has been much interest in the homonuclear systems [1-9], where the exchange energy is zero at infinite separation but, more significantly, decays exponentially with $R$. For sufficiently large $R$, it becomes impractical to merely subtract the energies of the two states considered. In addition, the preceding perturbation expansion is based on separate states at infinite separation, whereas in the homonuclear case the states are not localized on a particular atom for any value of $R$, however large. To overcome these problems, the Herring-Holstein result [7-9] is usually used, which expresses the exchange energy essentially as an integral over the median plane between the atoms together with a normalization term. This special case can be treated using the theory for heteronuclear systems by calculating $\psi_{A}$, the solution describing one of the atoms

\footnotetext{
*B.L.Burrows@staffs.ac.uk

†adalgarno@cfa.harvard.edu

${ }^{\ddagger}$ maurice@fh.huji.ac.il
}

perturbed by the other atom. In a later section of this article, exchange energies for several alkali-metal molecular ions are calculated for a large range of $R$.

\section{HYDROGENLIKE HETERONUCLEAR SYSTEMS}

The basic, exact, nonrelativistic Schrödinger equation for the interaction of two atoms is

$$
\left(-\frac{1}{2} \nabla^{2}-\frac{\mu_{a}}{r_{a}}-\frac{\mu_{b}}{r_{b}}\right) \Psi=E \Psi .
$$

Here $\mu_{a}, \mu_{b}$ are the charges on the atoms and the eigenvalue $E$ is given by

$$
E=W-\frac{\mu_{a} \mu_{b}}{R},
$$

where $W$ is the exact energy. Equation (1) separates into spheroidal coordinates:

$$
p=\frac{r_{a}+r_{b}}{R}, \quad q=\frac{r_{a}-r_{b}}{R}, \quad \Phi .
$$

We consider here only those states for which the azimuthal quantum number is zero and write $\Psi=X(p) Y(q)$ to obtain

$$
\begin{aligned}
& \frac{d}{d p}\left\{\left(p^{2}-1\right) \frac{d X}{d p}\right\}+\left[-C-\lambda^{2}\left(p^{2}-1\right)\right. \\
& \left.+R\left(\mu_{a}+\mu_{b}\right) p\right] X=0, \\
& \frac{d}{d q}\left\{\left(1-q^{2}\right) \frac{d Y}{d q}\right\}+\left[C-\lambda^{2}\left(1-q^{2}\right)\right. \\
& \left.-R\left(\mu_{a}-\mu_{b}\right) q\right] Y=0
\end{aligned}
$$

for $1 \leqslant p<\infty,-1 \leqslant q \leqslant 1$, where $C$ is the separation constant and

$$
\lambda=R \sqrt{-\frac{E}{2}} .
$$

Equations (4) and (5) have the same essential form and may be treated analogously using transformations similar to those in $[10,11]$. In Eq. (4) we may use $x=2 \lambda(p-1)$ and define $S=R\left(\mu_{a}+\mu_{b}\right) / \lambda$ so that, asymptotically, $S$ is independent 
of $R$ (it depends on $R$ through the dependence of $E$ on $R$ ). This leads to

$$
\begin{aligned}
(x+ & \left.\frac{x^{2}}{4 \lambda}\right) \frac{d^{2} X}{d x^{2}}+\left(1+\frac{x}{2 \lambda}\right) \frac{d X}{d x} \\
& +\left(-\frac{C}{4 \lambda}-\frac{x}{4}-\frac{x^{2}}{16 \lambda}+\frac{S}{4}+\frac{S x}{8 \lambda}\right) X=0 .
\end{aligned}
$$

Writing $X=\exp (-x / 2) \phi=\exp [\lambda(p-1)] \phi$, we may rearrange Eq. (7) into a form suitable for perturbation theory:

$$
\begin{gathered}
L(\gamma, 1, x) \phi=-\omega x L(s, 2, x) \phi, \\
L(a, b, x)=x D^{2}+(b-x) D-a,
\end{gathered}
$$

where

$$
D=\frac{d}{d x}, \quad \omega=\frac{1}{4 \lambda}, \quad \gamma=\omega C-\frac{S}{4}+\frac{1}{2}, \quad s=1-\frac{S}{2},
$$

so that $\omega \sim 1 / R$ is a natural parameter for perturbation theory.

Asymptotically, we may neglect the right-hand side of Eq. (8) and obtain exact solutions to the resulting confluent hypergeometric equation. Only the regular Kummer $M$ solution, $M(\gamma, 1, x)$, need be considered, since we require solutions which are regular at $x=0$. To ensure a physical solution also as $x \rightarrow \infty$, we require $\gamma=-n$, where $n$ is zero or a positive integer, leading to un-normalized solutions of the form

$$
\phi=M(\gamma, 1, x)
$$

For the ground state, $\gamma=n=0$, and Eqs. (8) and (9) then yield

$$
\phi_{0}=1, \quad \frac{C}{4 \lambda}=\left(\frac{S}{4}-\frac{1}{2}\right) .
$$

To improve these results, we now expand $\phi$ and $C$ in power series in $\omega$, and use algebraic perturbation theory (PT). We introduce a standard realization of the generators of the $\mathrm{SO}(2,1)$ Lie algebra,

$$
J_{-}=x D^{2}+D, \quad J_{0}=x D+\frac{1}{2}, \quad J_{+}=x,
$$

which satisfy the usual commutation relationships

$$
\left[J_{0}, J_{+}\right]=J_{+}, \quad\left[J_{0}, J_{-}\right]=-J_{-}, \quad\left[J_{+}, J_{-}\right]=-2 J_{0},
$$

so that Eq. (8) becomes

$$
\begin{aligned}
& {\left[J_{-}-J_{0}-\left(\gamma-\frac{1}{2}\right)\right] \phi} \\
& \quad=-\omega\left\{J_{0}-\frac{1}{2}+J_{+}\left[J_{-}-J_{0}+\frac{1}{2}-s\right]\right\} \phi .
\end{aligned}
$$

We first apply a similarity transformation (often called a "rotation" [12,13]) to Eq. (14) so that, for a general operator $A$,

$$
A \phi=0 \rightarrow B \psi=0,
$$

where $(B, \psi)$ are transforms of $(A, \phi)$ defined by

$$
B=\hat{U} A \hat{U}^{-1}, \quad \psi=\hat{U} \phi .
$$

With the particular choice

$$
\hat{U}=\exp \left(J_{-}\right)
$$

we have

$$
J_{0} \rightarrow J_{0}+J_{-}, \quad J_{+} \rightarrow\left(J_{+}+2 J_{0}+J_{-}\right),
$$

and the complete Eq. (8) is transformed by Eqs. (16) and (17) to

$$
h \psi=\left(h_{0}+\omega h_{1}\right) \psi=\gamma \psi
$$

where

$$
\begin{gathered}
h_{0}=\left(\frac{1}{2}-J_{0}\right), \\
h_{1}=\left(J_{+}+2 J_{0}+J_{-}\right)\left(b+h_{0}\right)+\left(J_{-}-h_{0}\right), \\
b=\frac{S}{2}-1,
\end{gathered}
$$

and we now expand $(\psi, \gamma)$ in a power series in $\omega$. Since $\psi_{0}=$ $\hat{U} \phi_{0}$ for the correct behavior at infinite separation, we can proceed by ordinary PT, constructing the matrices for the $J$ operators in the basis $\left\{x^{n}, n=0,1,2 \ldots\right\}$ so that $n$ is a nonnegative integer and arrive at the following matrix form of Eq. (19):

$$
H_{0} \mathbf{x}=\left(\gamma-\omega H_{1}\right) \mathbf{x}
$$

We give a simple example of the matrix formulation in Appendix A.

If we now expand $\gamma$ and the vector $\mathbf{x}$ in powers of $\omega$, we obtain for the ground state a conventional perturbation scheme beginning with $\gamma_{0}=0$ and $\mathbf{x}_{\mathbf{0}}{ }^{T}=(1,0,0,0, \ldots)$ corresponding to the asymptotic (zero-order) solution. The higher equations may be made consistent order by order through appropriate choices of the sucessive $\gamma_{n}$. These are polynomials in $b$ and consequently functions of $\lambda$ and hence of $E$. This is an elementary, but tedious, calculation, but which can be carried out quickly and straightforwardly using a standard symbolic mathematical package; here we have used MAPLE. Further details and an example of the operators involved and some of the polynomials obtained are given in Appendix A. We note that since $J_{ \pm}$merely connect a basis element with an adjacent element, $\mathrm{H}_{1}$ has a band structure whereby in the perturbation calculation, one new basis element is introduced at each order of perturbation.

Equation (5) can be treated analogously, choosing either $y=2 \lambda(1+q)$ appropriate for a function centered (localized) on atom $A$ or $2 \lambda(1-q)$ for localization on atom $B$ at infinite separation. In either case, the algebraic treatment is based on the realization of the $\mathrm{SO}(2,1)$ generators (dependent on $y$ and $D=d / d y$ instead of $x$ and $D=d / d x$ ) but is completely analogous to the preceding analysis. Here the appropriate basis consists of integral powers of $y$. We note that the terms in $C$ in Eqs. (4) and (5) are of opposite sign, as a result of which the sign of $\omega$ is reversed in the analog of Eq. (8):

$$
L(\delta, 1, y) \psi=\omega y L(t, 2, y)
$$

where now

$$
D=\frac{d}{d y}, \quad \delta=-\omega C+\frac{t}{2}, \quad t=1-\frac{T}{2}, \quad T=\frac{R\left(\mu_{a}-\mu_{b}\right)}{\lambda} .
$$


TABLE I. Energies for the $1 s \sigma$ state in $\mathrm{HeH}^{2+}$ using $n$ th-order perturbation theory (PTn) (a.u.).

\begin{tabular}{lcccc}
\hline \hline$R$ & PT2 & PT3 & PT4 & Exact \\
\hline 3 & -1.6661 & -1.6675 & -1.6681 & -1.6688 \\
4 & -1.7497 & -1.7503 & -1.7505 & -1.7506 \\
5 & -1.7998 & -1.8001 & -1.8002 & -1.8002 \\
6 & -1.8332 & -1.8334 & -1.8334 & \\
8 & -1.8750 & -1.8750 & -1.8750 & \\
10 & -1.9000 & -1.9000 & -1.9000 & \\
50 & -1.9800 & -1.9800 & -1.9800 & \\
\hline \hline
\end{tabular}

The two perturbation expansions calculated through $m$ th order for the separation constant $C$ depend finally only on $\lambda$, so that for any given (finite) $R$, we need to solve the equation

$$
\sum_{j=0}^{m} \gamma^{j} \omega^{j}=\sum_{j=0}^{m} \delta^{j}(-\omega)^{j}
$$

to obtain estimates of $E$. In each case the basis needs to be truncated, but we can ensure that it is sufficiently large not to affect the perturbation expansion since the zero-order function is 1 and the band structure of the matrices is such that only one extra basis function is needed for each order of perturbation. Thus, for $m$ th-order perturbation the size of the matrices required is $m+1$ and the calculations involved are essentially matrix multiplications together with the solution of the transcendental equation (24).

To examine this technique, we consider $\mathrm{HeH}^{2+}\left(\mu_{a}=2\right.$, $\mu_{b}=1$ ), for which we have comparison data for values of $R$ from $3 \ldots 5[14,15]$, and the exchange energy at infinite separation is -1.5 . The two states considered are the $1 s \sigma$ and $2 p \sigma$ states, and it is well known that perturbation expansions for the latter are troublesome since it has the same energy at infinite separation as the $2 s \sigma$ and $3 d \sigma$ states. The results are given in Tables I and II (in atomic units).

The results for the $1 s \sigma$ state are excellent even for low values of $R$, but for the $2 p \sigma$ state they are less good for the smaller values of $R$. The form of $\mathrm{H}_{1}$ in Eq. (17) is dominated by the matrix $J_{-} h_{0}$ and the maximum size of the elements increases as the order of the perturbation increases so that there are ranges of $\omega$ for which we have convergence and ranges where the perturbation expansion is asymptotic; for $R \approx 3$, in the case of $2 p \sigma$, the perturbation is large, so an asymptotic expansion is likely. In the perturbation calculations in this article, we have chosen the higher-order perturbed vectors, $\mathbf{x}_{\mathbf{n}}$, to have a zero component in $\mathbf{x}_{\mathbf{0}}$. This is not strictly necessary

TABLE II. Energies for the $2 p \sigma$ state in $\mathrm{HeH}^{2+}$ using $\mathrm{PT} n$ (a.u.).

\begin{tabular}{lcccc}
\hline \hline$R$ & PT2 & PT3 & PT4 & Exact \\
\hline 3 & -0.4736 & -0.5045 & -0.5279 & -0.5126 \\
4 & -0.4847 & -0.5012 & -0.5114 & -0.5315 \\
5 & -0.4903 & -0.5003 & -0.5056 & -0.5225 \\
6 & -0.4934 & -0.5000 & -0.5030 & \\
8 & -0.4966 & -0.5000 & -0.5011 & \\
10 & -0.4980 & -0.5000 & -0.5005 & \\
50 & -0.5000 & -0.5000 & -0.5000 & \\
\hline \hline
\end{tabular}

and there are free parameters in the PT. We hope to return to this problem in future work using the free parameters to improve the convergence properties of the perturbation calculation. One possibility for $\mathrm{HeH}^{2+}$ is to ensure orthogonality between the $1 s \sigma$ and $2 p \sigma$ states to all orders. However, the technique here was designed for large $R$, and for these larger values the lower-order perturbation results give consistent estimates.

\section{GENERAL HETERONUCLEAR SYSTEMS}

For these systems we have the Schrödinger equation

$$
\left[-\frac{1}{2} \nabla^{2}+V_{A}\left(r_{a}\right)+V_{B}\left(r_{b}\right)-\frac{1}{r_{a}}-\frac{1}{r_{b}}\right] \Psi=E \Psi,
$$

where $V_{A}$ and $V_{B}$ are core potentials which die away fairly rapidly so that the system may be treated in the same way essentially in a region well away from either atom (where both $V_{A}$ and $V_{B}$ can be ignored) with $\mu_{a}=\mu_{b}=1$, but the solution at infinite separation is different and needs closer analysis. We consider a state localized on atom $A$ in a region where $V_{A} \approx V_{B} \approx 0$ and with a large internuclear separation so that $1 / r_{b}$ may be replaced by $1 / R$. We have a zero-order equation of the form

$$
\left(-\frac{1}{2} \nabla^{2}-\frac{1}{r_{a}}\right) \Psi_{a}=E_{a} \Psi_{a},
$$

with $E_{a}$ approximately equal to the ionization energy of the valence electron on atom $A$. For fixed $E_{a}=-\beta^{2} / 2$ a solution of this hydrogenlike system, which vanishes as $r_{a} \rightarrow \infty$, may be written in the form

$$
\begin{gathered}
\Psi_{a}^{0}=\exp \left(-\beta r_{a}\right) U\left(c, 2,2 \beta r_{a}\right) \sim \exp \left(-\beta r_{a}\right)\left(2 \beta r_{a}\right)^{-c}, \\
c=1-\frac{1}{\beta},
\end{gathered}
$$

where $U$ denotes the Kummer $U$ function. In the case where $E_{a}$ is exactly equal to the ionization energy, this will be the required, spherically symmetric, solution at infinite separation. However, for large finite $R$, which corresponds to our zeroorder situation, we have degenerate solutions of the equation in the form

$$
\begin{aligned}
\Psi_{a}^{l} & =\exp \left(-\beta r_{a}\right) r_{a}^{l} P_{l}[\cos (\theta)] U\left(c+l, 2 l+2,2 \beta r_{a}\right) \\
& \sim(2 \beta)^{-l} \Psi_{a}^{0} P_{l}[\cos (\theta)]
\end{aligned}
$$

and the required zero-order wave function is a linear combination of all of these solutions. This is examined in Appendix B, where it is shown that the spheroidal coordinates we are using are asymptotically equivalent to parabolic coordinates and using these coordinates we obtain for large $R$ an appropriate one-centre wave function centered on atom $A$ :

$$
\begin{aligned}
\Psi_{a}= & \exp [-2 R \alpha(p-1)] \exp [-2 R \alpha(q+1)] \\
& \times U[d, 1,2 R \alpha(q+1)], \\
& \alpha=\sqrt{-\frac{E_{a}}{2}}, \quad d=1-\frac{1}{2 \alpha} .
\end{aligned}
$$

\section{A. Algebraic perturbation theory}

The form of the asymptotic wave function is separable in $p$ and $q$ and of identical form in $p$ to that for the hydrogenlike 
systems. Thus, the analysis of Eq. (4) is unchanged. The asymptotic solution for Eq. (5) has an additional factor $U[d, 1,2 \lambda(q+1)]$, where

$$
\begin{aligned}
U(d, 1, y) & =y^{-d}\left[1-\frac{d^{2}}{y}+\frac{d^{2}(d+1)^{2}}{2 ! y^{2}}-\cdots\right] \\
& =\exp \left(-J_{-}\right) y^{-d}
\end{aligned}
$$

for large $y$. Thus, Eq. (5) can be analyzed analogously to the case of the hydrogenlike system and we have the algebraic perturbation equation,

$$
h_{0} \psi=\omega\left(\left(J_{+}+2 J_{0}+J_{-}\right)\left(b+h_{0}\right)+\left(J_{-}-h_{0}\right)\right) \psi+\delta \psi,
$$

where $T=0$ so that $b=-1$. The required asymptotic solution of this equation is $y^{-d}$ so that we may use the basis $\left\{y^{-d+n}\right\}$, where now $n$ is any integer, positive or negative, and since

$$
h_{0} y^{-d}=-d y^{-d}
$$

we have

$$
\delta_{0}=\frac{1}{2}-d
$$

Note that the negative values of $n$ did not occur in the hydrogenlike heteronuclear systems since for these $J_{-} k=0$ for any constant $k$, but in the general case this is no longer true. In all other respects the calculation is identical to the calculation for the hydrogenlike atoms and the band form of the perturbation matrices ensures that we only have two extra basis functions for each order of perturbation (one new positive value of $\mathrm{n}$ and one new negative value) and that consequently the basis can be truncated appropriately. In Tables III and IV the first-, second-, third-, and eighth-order perturbation results are presented for both $\mathrm{Li}$ and $\mathrm{Na}$ perturbed by $-\frac{1}{r_{b}}$ for a wide range of nuclear separations. Assuming $R$ is sufficiently large so that in the region considered the core potentials are negligible and not affected by the other atom, then these solutions may be used as a model for the composite molecule $\mathrm{Li}-\mathrm{Na}$, and the exchange energies are obtained by subtracting the energies of the localized state. Thus, analogously to $\mathrm{HeH}^{2+}$, we obtain two states localized on the different atoms. In homonuclear systems, and large $R$, subtracting two energies may not be a practical procedure and we need to examine the alternative approach using the Herring-Holstein theory.

TABLE III. Energies of perturbed Li using PTn (a.u.).

\begin{tabular}{lcccc}
\hline \hline$R$ & PT1 & PT2 & PT3 & PT8 \\
\hline 5 & -0.19507 & -0.22242 & -0.23549 & -0.25338 \\
10 & -0.19656 & -0.20526 & -0.20724 & -0.20780 \\
20 & -0.19761 & -0.20021 & -0.20051 & -0.20052 \\
30 & -0.19789 & -0.19914 & -0.19923 & -0.19923 \\
40 & -0.19800 & -0.19873 & -0.19878 & -0.19878 \\
50 & -0.19806 & -0.19854 & -0.19856 & -0.19856 \\
75 & -0.19812 & -0.19834 & -0.19835 & -0.19835 \\
100 & -0.19814 & -0.19827 & -0.19827 & -0.19827 \\
$\infty$ & -0.198174 & -0.198174 & -0.198174 & -0.198174 \\
\hline \hline
\end{tabular}

TABLE IV. Energies of perturbed Na using PTn (a.u.).

\begin{tabular}{llllc}
\hline \hline$R$ & \multicolumn{1}{c}{ PT1 } & PT2 & \multicolumn{1}{c}{ PT3 } & PT8 \\
\hline 5 & -0.18756 & -0.21540 & -0.22826 & -0.24618 \\
10 & -0.18821 & -0.19709 & -0.19907 & -0.19963 \\
20 & -0.18905 & -0.19171 & -0.19202 & -0.19204 \\
30 & -0.18930 & -0.19057 & -0.19067 & -0.19068 \\
40 & -0.18940 & -0.19015 & -0.119019 & -0.19019 \\
50 & -0.118944 & -0.18994 & -0.18996 & -0.18996 \\
75 & -0.18950 & -0.18973 & -0.18973 & -0.18974 \\
100 & -0.18952 & -0.18965 & -0.18966 & -0.18966 \\
$\infty$ & -0.189552 & -0.189552 & -0.189552 & -0.189552 \\
\hline \hline
\end{tabular}

\section{THEORY OF HOMONUCLEAR HYDROGENLIKE SYSTEMS}

The theory cannot be directly applied to homonuclear systems since for such systems, at infinite separation we do not have localization on one particular atom. The lowest states are the gerade and ungerade states, which are respectively symmetric and antisymmetric with respect to the median plane $(z=R / 2)$ between the two identical atoms. These two states, $\psi_{+}$and $\psi_{-}$, can always be written in the form

$$
\psi_{+}=\frac{\psi_{A}+\psi_{B}}{\sqrt{2}}, \quad \psi_{-}=\frac{\psi_{A}-\psi_{B}}{\sqrt{2}},
$$

where $\psi_{A}, \psi_{B}$ are states localized on atoms $A$ and $B$, respectively. By symmetry considerations, on the median plane $\psi_{A}, \psi_{B}$ satisfy

$$
\psi_{A}=\psi_{B}, \quad \frac{\partial \psi_{A}}{\partial z}=-\frac{\partial \psi_{B}}{\partial z},
$$

and they can be obtained using the theory outlined previously, where one atom is perturbed by the other. In practice, using the symmetry, only one of the functions is required.

The exchange energy may be estimated from the HerringHolstein theory, which is an application of Green's theorem for two states, which satisfy

$$
\left(-\frac{1}{2} \nabla^{2}+V\right) \psi_{+}=E_{+} \psi_{+}, \quad\left(-\frac{1}{2} \nabla^{2}+V\right) \psi_{-}=E_{-} \psi_{-},
$$

from which we may deduce

$$
\begin{aligned}
& \left(E_{+}-E_{-}\right) \int_{\hat{V}} \psi_{+} \psi_{-} d \hat{V} \\
& \quad=-\frac{1}{2}\left[\int_{\hat{V}}\left(\psi_{-} \nabla^{2} \psi_{+}-\psi_{+} \nabla^{2} \psi_{-}\right) d \hat{V}\right] \\
& \quad=\int_{S} \psi_{A} \nabla \psi_{A} \cdot \mathbf{k} d S,
\end{aligned}
$$

where $S$ is the median plane and $\hat{V}$ the spatial volume $z<R / 2$. In terms of spheroidals, the median plane corresponds to $q=0$ and

$$
\nabla \cdot \mathbf{k}=\frac{2}{R} \frac{\partial}{\partial q}
$$


Thus the surface integral is

$$
R \pi \int_{p=1}^{\infty} \psi_{A} \frac{\partial \psi_{A}}{\partial q} d p
$$

The volume integral may also be expressed in these coordinates and is

$$
\left(\frac{R}{2}\right)^{3} \pi \int_{q=-1}^{q=0} \int_{p=1}^{\infty}\left(\psi_{A}^{2}-\psi_{B}^{2}\right)\left(p^{2}-q^{2}\right) d p d q,
$$

where $\psi_{B}$ may be calculated from $\psi_{A}$, but it is more consistent to ignore $\psi_{B}^{2}$ in this interval since it is $O[\exp (-2 R)]$ and terms of this order in $\psi_{A}^{2}$ cannot be obtained from our perturbation expansion, which is essentially in inverse powers of $R$. In order to examine these estimates, we have used the preceding theory to calculate the localized $\psi_{A}$ for $\mathrm{H}-\mathrm{H}$ at various values of $R$ for which exact results are known. These are computed to eighthorder perturbation and used in a Herring-Holstein calculation for the exchange energies. These are given in Table $\mathrm{V}$, where the well known Herring-Holstein asymptotic estimate is also given.

The perturbation results are universally better than the conventional asymptotic results. The calculation process, even for eighth-order perturbation, is fairly trivial since it only involves multiplication by matrices of low order. (In this latter calculation the basis was such that the matrices were of order $9 \times 9$.) These matrix calculations are exact and the only numerical aspect is the solution of the transcendental equation (24) for the separation constant (the accuracy of this solution will clearly affect the accuracy of the results) and the truncation to the order of the perturbation used.

From these results we obtain an analytical expansion of the exchange energy in terms of $R$. To do this we first fit the parameter $\alpha$ of Eq. (29) to a set of the numerical results, obtaining

$$
\alpha \approx 0.5+\frac{0.5}{R}-\frac{0.2410}{R^{2}} .
$$

TABLE V. Exchange energies for $\mathrm{H}_{2}{ }^{+}$: Eighth-order perturbation theory (PT8) compared with the exact and asymptotic values (a.u.).

\begin{tabular}{lccc}
\hline \hline$R$ & Asymptotic & PT8 & Exact \\
\hline 4 & 0.1078 & 0.09391 & 0.1005 \\
6 & 0.02188 & 0.02140 & 0.02136 \\
7 & 0.00939 & 0.00933 & 0.00932 \\
8 & 0.00395 & 0.00396 & 0.00399 \\
9 & 0.001634 & 0.001648 & 0.001652 \\
10 & 0.000668 & 0.000676 & 0.000677 \\
11 & 0.000270 & 0.000274 & 0.000275 \\
12 & 0.000108 & 0.000110 & 0.000114 \\
13 & $4.32 \times 10^{-5}$ & $4.39 \times 10^{-5}$ & $4.40 \times 10^{-5}$ \\
14 & $1.71 \times 10^{-5}$ & $1.74 \times 10^{-5}$ & $1.74 \times 10^{-5}$ \\
15 & $6.76 \times 10^{-6}$ & $6.85 \times 10^{-6}$ & $6.88 \times 10^{-6}$ \\
20 & $6.07 \times 10^{-8}$ & $6.15 \times 10^{-8}$ & $6.17 \times 10^{-8}$ \\
30 & $4.13 \times 10^{-12}$ & $4.17 \times 10^{-12}$ & $4.19 \times 10^{-12}$ \\
\hline \hline
\end{tabular}

We used the values $R=80, R=90$, and $R=100$ to obtain this expression. The analysis can then be done exactly using MAPLE and we obtain the exchange energy

$$
\begin{aligned}
\Delta E= & R\left(3.98439+\frac{0.01559}{R}-\frac{10.70025}{R^{2}}-\frac{9.80912}{R^{3}} \cdots\right) \\
& \times \exp (-2 \alpha R) .
\end{aligned}
$$

By analyzing the exponential terms, this may be expressed in the more conventional form:

$$
\begin{aligned}
\Delta E= & R \exp (-R) \exp (-1) \\
& \times\left(1+\frac{0.48591}{R}-\frac{2.56749}{R^{2}}-\frac{3.73720}{R^{3}} \cdots\right) .
\end{aligned}
$$

This is similar, but not identical, to the usual asymptotic expansion.

\section{HOMONUCLEAR MOLECULES WITH CORE POTENTIALS}

We may also consider the general homonuclear case by analyzing Eq. (25) when the atomic cores on atoms $A$ and $B$ are identical. We may apply the theory outlined previously given an approximation for $d$ so that we can construct a basis. Taking $E_{a}$ to be the ionization energy in Eq. (29) provides an appropriate basis and the localized perturbation calculation can be carried out from either atom. One important difference in the perturbation calculation is that at each order of the perturbation two extra basis functions are used (so that for the initial function $y^{-d}$, first-order PT would lead to contributions from the basis elements $y^{-d+1}, y^{-d-1}$ ). We may calculate (38) as in the $\mathrm{H}-\mathrm{H}$ case since it depends only on the solution on the median plane, but we need to estimate the volume integral [Eq. (40)] from additional information about the atomic core. This is equivalent to finding a core-dependent normalization constant so that $\psi_{A}$ may be replaced by $k \psi_{a}$.

\section{A. Normalization}

This problem has been considered in previous work [1,2,6] using perturbation based on radial coordinates and the zeroorder wave function in the asymptotic region is taken to be Eq. (27) with $E_{a}$ given by the ionization energy. There are many ways to estimate the normalization constant $k[1,6]$ and all estimates seem to be consistent but using such a constant inevitably restricts the accuracy of the estimates of the exchange energies and leads to an uncertainty which may be estimated to be the order of $1 \%-3 \%$. One way is to compute the value and derivative of $\Psi_{a}^{0}$ at some $r=r_{0}$ and solve for the total wave function numerically using pseudopotentials for the atomic cores [16], obtaining $\Psi_{a}^{0}$ for all $r$. The approximate zero-order wave function obtained is spherically symmetric, and this leads to simplification when the integration is carried out over a spherical region. In principle, this can be done for all $\Psi_{a}^{l}$. Since most of the contribution to Eq. (40) comes from the atomic core and a small asymptotic region, we may estimate the volume integral by integrating over a large spherical volume encompassing these regions. Over such a volume the wave function may be approximated by $\Psi_{a}^{0}+\hat{\Psi}_{a}$, 
TABLE VI. Exchange energies for $\mathrm{Li}_{2}{ }^{+}$using PT8 (A) compared with variational calculations (B). In the rightmost column, the ratio between the two sets of numbers is given (a.u.).

\begin{tabular}{|c|c|c|c|}
\hline$R$ & A & B & Ratio \\
\hline 8 & 0.06477659 & 0.06465600 & 1.001865101 \\
\hline 8.5 & 0.05693189 & 0.05553420 & 1.025168095 \\
\hline 9 & 0.04892875 & 0.04733190 & 1.033737289 \\
\hline 9.5 & 0.04136680 & 0.04004778 & 1.032936158 \\
\hline 10 & 0.03453352 & 0.03364689 & 1.026351024 \\
\hline 10.5 & 0.02853717 & 0.02807448 & 1.016480804 \\
\hline 11 & 0.02338416 & 0.02326550 & 1.005100256 \\
\hline 11.5 & 0.01902532 & 0.01915056 & 0.9934602435 \\
\hline 12 & 0.01538394 & 0.01565938 & 0.9824102420 \\
\hline 12.5 & 0.01237263 & 0.01272270 & 0.9724853785 \\
\hline 13 & 0.00990339 & 0.01027350 & 0.9639743028 \\
\hline 13.5 & 0.00789320 & 0.00824811 & 0.9569707484 \\
\hline 14 & 0.00626690 & 0.00658668 & 0.9514505031 \\
\hline 14.5 & 0.00495837 & 0.00523422 & 0.9472987379 \\
\hline 15 & 0.00391060 & 0.00414105 & 0.9443492700 \\
\hline 15.5 & 0.00307525 & 0.00326313 & 0.9424233788 \\
\hline 16 & 0.00241185 & 0.00256214 & 0.9413420032 \\
\hline 16.5 & 0.00188687 & 0.00200530 & 0.9409415050 \\
\hline 17 & 0.00147275 & 0.00156497 & 0.9410723528 \\
\hline 17.5 & 0.00114706 & 0.00121818 & 0.9416178233 \\
\hline 18 & 0.00089160 & 0.00094603 & 0.9424648267 \\
\hline 18.5 & 0.00069173 & 0.00073313 & 0.9435297969 \\
\hline 19 & 0.00053572 & 0.00056705 & 0.9447491403 \\
\hline 19.5 & 0.00041421 & 0.00043781 & 0.9460953382 \\
\hline 20. & 0.00031976 & 0.00033748 & 0.9474931848 \\
\hline 20.5 & 0.00024648 & 0.00025975 & 0.9489124158 \\
\hline 21 & 0.00018973 & 0.00019964 & 0.9503606492 \\
\hline 21.5 & 0.00014586 & 0.00015324 & 0.9518402506 \\
\hline 22 & 0.00011198 & 0.00011748 & 0.9531835206 \\
\hline 22.5 & 0.00008588 & 0.00008996 & 0.9546465096 \\
\hline 23 & 0.00006578 & 0.00006881 & 0.955965707 \\
\hline 23.5 & 0.00005033 & 0.00005258 & 0.9572080639 \\
\hline 24 & 0.00003847 & 0.00004013 & 0.9586344381 \\
\hline 24.5 & 0.00002938 & 0.00003060 & 0.9601307190 \\
\hline 25 & 0.00002241 & 0.00002331 & 0.9613899614 \\
\hline 25.5 & 0.00001708 & 0.00001774 & 0.9627959414 \\
\hline 27 & 0.00000753 & 0.00000778 & 0.9678663239 \\
\hline 26. & 0.00001301 & 0.00001349 & 0.9644180875 \\
\hline 26.5 & 0.00000990 & 0.00001025 & 0.9658536585 \\
\hline 27.5 & 0.00000572 & 0.00000590 & 0.9694915254 \\
\hline 28 & 0.00000434 & 0.00000447 & 0.9709172260 \\
\hline 28.5 & 0.00000329 & 0.00000338 & 0.9733727811 \\
\hline 29 & 0.00000250 & 0.00000255 & 0.980392159 \\
\hline 30 & 0.00000143 & 0.00000145 & 0.9862068966 \\
\hline 31 & $8.190333400 \times 10^{-7}$ & $8.468815650 \times 10^{-7}$ & 0.9671167420 \\
\hline 32 & $4.675271850 \times 10^{-7}$ & $4.829070270 \times 10^{-7}$ & 0.9681515465 \\
\hline 33 & $2.663025430 \times 10^{-7}$ & $2.747848940 \times 10^{-7}$ & 0.9691309414 \\
\hline 35 & $8.588708750 \times 10^{-8}$ & $8.845697500 \times 10^{-8}$ & 0.9709475991 \\
\hline 37 & $2.750188780 \times 10^{-8}$ & $2.827647290 \times 10^{-8}$ & 0.9726067285 \\
\hline 39 & $8.750160620 \times 10^{-9}$ & $8.982464200 \times 10^{-9}$ & 0.9741381012 \\
\hline 40 & $4.924821370 \times 10^{-9}$ & $5.051807900 \times 10^{-9}$ & 0.9748631515 \\
\hline
\end{tabular}

where $\hat{\Psi}_{a}$ is a combination of functions of the form given in Eq. (28) and these are orthogonal to $\Psi_{a}^{0}$ over this volume. Consequently, to lowest order, we may simply renormalize $\Psi_{a}^{0}$ so that the volume integral is unity. To use this, we need to
TABLE VII. Exchange energies for $\mathrm{Li}_{2}{ }^{+}$using PT8 (A) compared with pseudopotential calculations (B) (a.u.).

\begin{tabular}{lcc}
\hline \hline$R$ & $\mathrm{~A}$ & $\mathrm{~B}$ \\
\hline 20 & $3.20 \times 10^{-4}$ & $3.34 \times 10^{-4}$ \\
30 & $1.43 \times 10^{-6}$ & $1.46 \times 10^{-6}$ \\
40 & $4.92 \times 10^{-9}$ & $4.96 \times 10^{-9}$ \\
50 & $1.47 \times 10^{-11}$ & $1.47 \times 10^{-11}$ \\
60 & $4.02 \times 10^{-14}$ & $4.02 \times 10^{-14}$ \\
70 & $1.04 \times 10^{-16}$ & $1.03 \times 10^{-16}$ \\
80 & $2.55 \times 10^{-19}$ & $2.54 \times 10^{-19}$ \\
90 & $6.06 \times 10^{-22}$ & $6.05 \times 10^{-22}$ \\
\hline \hline
\end{tabular}

normalize our solution in Eq. (29) in the same way as $R \rightarrow \infty$ and it suffices to ensure this using the $z$ axis $(p=1)$ so that we need to choose

$$
k=\frac{\hat{q}}{\sqrt{4 \pi}} \frac{1}{(2 \beta)^{d}},
$$

where $\hat{q}$ is taken from the numerical calculation or from previous estimates. In order to compare with some previous calculations we have taken the values from [2] and these are given in Appendix $\mathrm{C}$, together with the other parameters used for the ions $\mathrm{Li}_{2}{ }^{+}, \mathrm{Na}_{2}{ }^{+}, \mathrm{K}_{2}{ }^{+}, \mathrm{Rb}_{2}{ }^{+}$, and $\mathrm{Cs}_{2}{ }^{+}$. In Table VI we compare our estimates for the exchange energies in $\mathrm{Li}_{2}{ }^{+}$ (A) with some variational calculations (B) [17] and note that there is good agreement. The variational calculations are more challenging for the larger values of $R$ where current interest lies and in Table VII we compare our estimates for larger $R$ with results obtained in [2]. Finally, in Table VIII we give our estimates for the exchange energies of the other systems. We note that in the earlier work of Bardsley et al. [1], the exchange energies were estimated using a two-term asymptotic expansion. Even though the parameters used were slightly different the results obtained are consistent with our values.

The results quoted are evaluated from analytical formulas obtained for the exchange energies, analogously to Eq. (42) for $\mathrm{H}_{2}{ }^{+}$, rather than the slightly more accurate numerical results. As in the construction of Eq. (41) it is first necessary to fit $\alpha$ to a set of the energies and then we obtain a formula of the form

$$
\Delta E=k(\alpha R)^{-2 d} R \exp (-2 \alpha R)\left(A+\frac{B}{R}+\frac{C}{R^{2}} \cdots\right)
$$

TABLE VIII. Exchange energies for $\mathrm{Na}_{2}{ }^{+}, \mathrm{K}_{2}{ }^{+}, \mathrm{Rb}_{2}{ }^{+}$, and $\mathrm{Cs}_{2}{ }^{+}$ using PT8 analytically using a fit to $\alpha$ (a.u.).

\begin{tabular}{ccccc}
\hline \hline$R$ & $\mathrm{Na}-\mathrm{Na}$ & $\mathrm{K}-\mathrm{K}$ & $\mathrm{Rb}-\mathrm{Rb}$ & $\mathrm{Cs}-\mathrm{Cs}$ \\
\hline 10 & $4.03 \times 10^{-2}$ & $5.99 \times 10^{-2}$ & $6.73 \times 10^{-2}$ & $1.57 \times 10^{-1}$ \\
15 & $5.04 \times 10^{-3}$ & $1.22 \times 10^{-2}$ & $1.48 \times 10^{-2}$ & $2.03 \times 10^{-2}$ \\
20 & $4.50 \times 10^{-4}$ & $1.57 \times 10^{-3}$ & $2.08 \times 10^{-3}$ & $3.38 \times 10^{-3}$ \\
30 & $2.38 \times 10^{-6}$ & $1.57 \times 10^{-5}$ & $2.40 \times 10^{-5}$ & $5.07 \times 10^{-5}$ \\
40 & $9.60 \times 10^{-9}$ & $1.15 \times 10^{-7}$ & $1.99 \times 10^{-7}$ & $5.29 \times 10^{-7}$ \\
50 & $3.35 \times 10^{-11}$ & $7.10 \times 10^{-10}$ & $1.39 \times 10^{-9}$ & $4.59 \times 10^{-9}$ \\
60 & $1.07 \times 10^{-13}$ & $3.96 \times 10^{-12}$ & $8.75 \times 10^{-12}$ & $3.57 \times 10^{-11}$ \\
70 & $3.18 \times 10^{-16}$ & $2.06 \times 10^{-14}$ & $5.12 \times 10^{-14}$ & $2.57 \times 10^{-13}$ \\
80 & $9.10 \times 10^{-19}$ & $1.02 \times 10^{-16}$ & $2.84 \times 10^{-16}$ & $1.75 \times 10^{-15}$ \\
90 & $2.51 \times 10^{-21}$ & $4.82 \times 10^{-19}$ & $1.51 \times 10^{-18}$ & $1.14 \times 10^{-17}$ \\
\hline \hline
\end{tabular}


by fitting at $R=90, R=100$, and $R=500$. The values of $A, B$, and $C$ and the expressions for $\alpha$ are given in Appendix $C$ for all the systems considered. The perturbation series for $\mathrm{Cs}$ is beginning to behave as an asymptotic series for $R \approx 10 \cdots 15$ and lower-order perturbation is preferable, but the fitted formula reproduces these lower-order estimates to $1 \%$ or $2 \%$. The results obtained show that the algebraic approach provides an accurate method for estimating exchange energies for a wide range of systems. In the case of homonuclear molecules, we show that it can be applied to $\mathrm{Li}_{2}{ }^{+}, \mathrm{Na}_{2}{ }^{+}, \mathrm{K}_{2}{ }^{+}$, $\mathrm{Rb}_{2}{ }^{+}$, and $\mathrm{Cs}_{2}{ }^{+}$and is not restricted to $\mathrm{H}_{2}{ }^{+}$. Further work is in progress, particularly on the hetronuclear systems that have been less widely studied.

\section{ACKNOWLEDGMENTS}

One of us (B.L.B.) thanks the Physical Chemistry Department at the Hebrew University of Jerusalem and the Institute of Atomic and Molecular Physics at Harvard for their hospitality and support and the EPSRC for the provision of Grant No. EP/F005199/1.

\section{APPENDIX A}

If we employ a finite basis $\left\{x^{n}\right.$ with $\left.n=0,1,2,3\right\}$, then the matrices $J_{0}, J_{+}, J_{-}$, and $\mathrm{H}_{1}$ are given by

$$
\begin{gathered}
J_{0}=\left(\begin{array}{cccc}
0.5 & 0 & 0 & 0 \\
0 & 1.5 & 0 & 0 \\
0 & 0 & 2.5 & 0 \\
0 & 0 & 0 & 3.5
\end{array}\right), \quad J_{+}=\left(\begin{array}{cccc}
0 & 0 & 0 & 0 \\
1 & 0 & 0 & 0 \\
0 & 1 & 0 & 0 \\
0 & 0 & 1 & 0
\end{array}\right) \\
J_{-}=\left(\begin{array}{cccc}
0 & 1 & 0 & 0 \\
0 & 0 & 4 & 0 \\
0 & 0 & 0 & 9 \\
0 & 0 & 0 & 0
\end{array}\right), \\
\mathrm{H}_{1}=\left(\begin{array}{cccc}
b & b & 0 & 0 \\
b & 3 b-2 & 4 b-4 \\
0 & b-1 & 5 b-8 & 9 b-18 \\
0 & 0 & b-2 & 7 b-18
\end{array}\right)
\end{gathered}
$$

We use the generic form of the perturbation equation given in Sec. II for either the $p$ or the $q$ equation:

$$
H_{0} \mathbf{x}=\left(k-\hat{\omega} H_{1}\right) \mathbf{x}, \quad \mathbf{x}_{\mathbf{0}}^{\mathbf{T}}=(1,0,0,0),
$$

with $H_{0}$ the matrix representation of $h_{0}$ and $\hat{\omega}= \pm \omega, k=\gamma, \delta$ depending on which of the $p$ or $q$ equations are used. The $n$ th-order vector $\mathbf{x}_{\mathbf{n}}(n \neq 0)$ is chosen to have no component in $\mathbf{x}_{\mathbf{0}}$ and the first-, second-, and third-order values of $\gamma$ are given by the polynomials $b, b^{2}$, and $2 b^{3}-2 b$, respectively. The definition of $b$ differs for the $p$ and $q$ equations (see Sec. II).

In the more general case of the core potentials, the initial vector will be of the form $\mathbf{x}_{\mathbf{0}}^{\mathbf{T}}=(\ldots 0,0,0,1,0,0,0 \ldots)$, depending on the number of basis elements used, and the polynomials will depend on $d$ in addition to $b$.

\section{APPENDIX B: COORDINATE SYSTEMS}

We may use parabolic coordinates at one center (atom) so that

$$
x=\sqrt{u v} \cos (\Phi), \quad y=\sqrt{u v} \sin (\Phi), \quad z=\frac{1}{2}(u-v) .
$$

We are interested in an arbitrary plane so that for $\sigma$ states, we may take $\Phi=0$ and from atom $A$

$$
r_{a}^{2}=u v+\frac{1}{4}(u-v)^{2}=\left(\frac{u+v}{2}\right)^{2}
$$

Thus,

$$
\begin{gathered}
r_{a}=\frac{u+v}{2}, \quad z_{a}=r_{a} \cos \left(\theta_{a}\right)=\frac{1}{2}(u-v) \Rightarrow \\
u_{a}=r_{a}\left[1+\cos \left(\theta_{a}\right)\right], \quad v_{a}=r_{a}\left[1-\cos \left(\theta_{a}\right)\right] .
\end{gathered}
$$

This has made a direct connection between parabolic and radial, one-center coordinates and we now seek to connect them, asymptotically, to spheroidals where atom $A$ is one of the foci. Since $u_{a}, v_{a}$ are orthogonal coordinates, then so are

$$
p=1+\frac{v_{a}}{R}, \quad q=-1+\frac{u_{a}}{R} .
$$

Thus,

$$
\left(1+\frac{v_{a}}{R}\right)+\left(-1+\frac{u_{a}}{R}\right)=\frac{2 r_{a}}{R}=p+q,
$$

where $p$ and $q$ satisfy the same relationship as the spheroidal coordinates. Also,

$$
\frac{R}{2}(p q+1)=\frac{R}{2}\left(\frac{u_{a}-v_{a}}{R}\right)+O\left(\frac{1}{R}\right) \sim z_{a}
$$

as $R \rightarrow \infty$, which shows that $p$ and $q$ approach the relationships satisfied by the spheroidal coordinates asymptotically. The relationships are the same from atom $B$, except we take

$$
p=1+\frac{v_{b}}{R}, \quad q=1-\frac{u_{b}}{R}
$$

and the $z$ coordinate measured from $B$ (in the direction of $A$ ) is such that

$$
\frac{R}{2}(-p q+1)=\frac{R}{2}\left(\frac{u_{b}-v_{b}}{R}\right)+O\left(\frac{1}{R}\right) \sim z_{b} .
$$

Thus, asymptotically, we have

$$
p \approx 1+\frac{v_{a}}{R} \approx 1+\frac{v_{b}}{R}, \quad q \approx-1+\frac{u_{a}}{R} \approx 1-\frac{u_{b}}{R} .
$$

We now consider atom $A$ at large separation so that the effect of atom $B$ can be ignored, but where $r_{a}$ is sufficiently large so that any core potential $V_{A}$ may be neglected:

$$
H_{0} \Psi=\left(-\frac{1}{2} \nabla^{2}-\frac{1}{r_{a}}\right) \Psi=E_{a} \Psi
$$

In parabolic coordinates we have

$$
\nabla^{2}=\frac{4}{u+v}\left\{L_{u}+L_{v}\right\}, \quad L_{x}=x D_{x}^{2}+D_{x}, \quad D_{x}=\frac{d}{d x},
$$


so that we have

$$
\left[L_{u}+L_{v}+1+\frac{E_{a}(u+v)}{2}\right] F_{0}(u) G_{0}(v)=0, \quad \Psi=F_{0} G_{0} .
$$

We may separate this equation in a variety of ways, and a separation convenient for our analysis is

$$
\begin{gathered}
\left(L_{u}+\frac{E_{a}}{2} u+1\right) F_{0}=k F_{0}, \\
\left(L_{v}+\frac{E_{a}}{2} v\right) G_{0}=-k G_{0} .
\end{gathered}
$$

In Eq. (B14) we may write $G_{0}=\exp (-\alpha v) g_{0}$, with $2 \alpha^{2}=$ $-E_{a}$ and obtain the equation for $g_{0}$ :

$$
\left[v D_{v}^{2}+(1-2 \alpha v) D_{v}+k-\alpha\right] g_{0}=0 .
$$

Substituting $s=2 \alpha v$ gives

$$
\left[s D_{s}^{2}+(1-s) D_{s}-c\right] g_{o}=0, \quad c=\frac{1}{2}-\frac{k}{2 \alpha} .
$$

This is a Kummer equation and since we require $g_{0}$ to be finite as $s \rightarrow 0$ we need to use the Kummer $M$ function and

$$
g_{0}=M(c, 1,2 \alpha v) .
$$

However, we also require the solution to be finite as $v \rightarrow \infty$ then the function must truncate to a polynomial; for the ground state, this implies that

$$
c=0 \Rightarrow k=\alpha .
$$

With this value of $k$ we may use $F_{0}=\exp (-\alpha u) f_{0}$ in Eq. (B12) to obtain

$$
\left[u D_{u}^{2}+(1-2 \alpha u) D_{u}+1-2 \alpha\right] f_{0}=0 .
$$

Putting $s=2 \alpha u$ gives the Kummer equation

$$
\left[s D_{s}^{2}+(1-s) D_{s}-d\right] f_{o}=0, \quad d=1-\frac{1}{2 \alpha} .
$$

In the region considered where $u$ is large (and well away from the origin) and $d$ is fixed, we require to use of the Kummer $U$ function to ensure the correct behavior for large $u \leqslant R$ so that we have

$$
f_{0}=U(d, 1,2 \alpha u)
$$

[In the case of the ground state of hydrogenlike atoms, we have $d=0$, but in that case $U(0,1,2 \alpha u)=M(0,1,2 \alpha u)=1$ and the analysis is then identical with the analysis of this case.]

Thus, we have found the complete one-center wave function from $A$ at large separation for a fixed $E_{a}$ and we have an approximate zero-order function for our perturbation procedure,

$$
\begin{aligned}
\psi_{a} \approx & \exp \left[-\alpha\left(u_{a}+v_{a}\right)\right] U\left(d, 1,2 \alpha u_{a}\right) \sim \exp [-\lambda(p-1)] \\
& \times \exp [-\lambda(q+1)] U(d, 1,2 \lambda(q+1)),
\end{aligned}
$$

where $\lambda=R \sqrt{-E_{a} / 2}$ and $E_{a}$ approaches the ionization energy for atom $A$ as $R \rightarrow \infty$.

\section{APPENDIX C}

1. For Li we use $d=0.5884065, \hat{q}=0.814596, A=2$, $B=-3.88834, C=-25.96465$, and the expansion for $\alpha$ is

$$
0.314807101+\frac{0.79432015}{R}-\frac{0.223527154}{R^{2}}
$$

2. For Na we use $d=0.624130, \hat{q}=0.67512, A=2$, $B=-4.30044, C=-23.72333$, and the expansion for $\alpha$ is

$$
0.307856900+\frac{0.8122126}{R}-\frac{0.225614003}{R^{2}} .
$$

3. For $\mathrm{K}$ we use $d=0.770436, \hat{q}=0.598446, A=$ 2, $B=-6.24031, C=-36.01288$, and the expansion for $\alpha$ is

$$
0.282415606+\frac{0.8857372}{R}-\frac{0.33669640}{R^{2}} .
$$

4. For Rb we use $d=0.804772, \hat{q}=0.569227, A=2$, $B=-6.75747, C=-36.69509$, and the expansion for $\alpha$ is

$$
0.2770420918+\frac{0.9032259}{R}-\frac{0.43459552}{R^{2}} .
$$

5. For Cs we use $d=0.869254, \hat{q}=0.509376, A=2$, $B=-7.79547, C=-46.17154$, and the expansion for $\alpha$ is

$0.267481791+\frac{0.93775775917}{R}-\frac{0.96809533445}{R^{2}}$.

The parameters $\hat{q}$ and $d$ are obtained from the explicit core potentials in [16].
[1] J. N. Bardsley, T. Holstein, B. R. Junker, and S. Sinha, Phys. Rev. A 11, 1911 (1975).

[2] M. J. Jamieson, A. Dalgarno, M. Aymar, and J. Tharamel, J. Phys. B 42, 095203 (2009).

[3] T. C. Scott, J. F. Babb, A. Dalgarno, and J. D. Morgan III, J. Chem. Phys. 99, 2841 (1993).

[4] T. C. Scott, J. F. Babb, A. Dalgarno, and J. D. Morgan III, Chem. Phys. Lett. 203, 175 (1993).

[5] K. T. Tang, J. P. Toennies, M. Wanshura, and C. L. Yiu, J. Chem. Phys. 46, 3746 (1992).

[6] K. T. Tang, J. P. Toennies, and C. L. Yiu, J. Chem. Phys. 94, 7266 (1991).

[7] T. Holstein, J. Phys. Chem. 56, 832 (1952).

[8] C. Herring, Rev. Mod. Phys. 34, 631 (1962).
[9] C. Herring and M. Flicker, Phys. Rev. 134, A362 (1964).

[10] J. D. Power, Phil. Trans. R. Soc. London A 274, 663 (1973).

[11] R. J. Damburg and R. K. H. Propin, J. Phys. B 1, 681 (1968).

[12] B. L. Burrows, M. Cohen, and T. Feldmann, J. Math. Phys. 35, 5572 (1994).

[13] B. L. Burrows, M. Cohen, and T. Feldmann, J. Phys. B 28, 4249 (1995).

[14] A. Dalgarno and A. L. Stewart, Proc. R. Soc. A 238, 276 (1956).

[15] D. R. Bates and T. R. Carson, Proc. R. Soc. A 234, 207 (1956).

[16] M. Marinescu, H. R. Sadeghpour, and A. Dalgarno, Phys. Rev. A 49, 982 (1994).

[17] P. Zhang, E. Bodo, and A. Dalgarno, J. Phys. Chem. A 113, 15085 (2009). 